\title{
Medición del índice tobillo brazo mediante palpación para el diagnóstico de enfermedad arterial periférica
}

\author{
Ankle-brachial index measured by palpation for the diagnosis of peripheral arterial disease
}

Migliacci R y col. Fam Pract. 2008 Aug; 25(4):228-32.

\section{Objetivo}

Comparar la medición del índice tobillo-brazo de presión arterial (ITBPA) mediante la palpación y mediante ecografía doppler para el diagnóstico de enfermedad arterial periférica (EAP) en pacientes con riesgo cardiovascular (CV) aumentado.

\section{Diseño}

Estudio prospectivo ciego llevado a cabo en 24 centros de atención primaria de Toscana, Italia. Participaron 240 pacientes de 55 a 65 años de edad con uno o dos factores de riesgo CV ó de 65 a 80 años sin factores de riesgo CV. Fueron excluidos aquellos con eventos $\mathrm{CV}$ isquémicos previos y diagnostico de EAP.

\section{Descripción de las pruebas y test de referencia}

EI ITBPA medido con ecografía doppler tiene $91 \%$ de sensibilidad y $86 \%$ de especificidad para detectar una estenosis de al menos $50 \%{ }^{1}$. Surge de realizar el cociente entre la presión arterial sistólica (PAS) de cada tobillo -escogiéndose el valor más alto entre la arteria pedia y la tibial posterior- y el de la PAS más elevada de cualquiera de las arterias braquiales. Un ITBPA mayor a 0,9 es considerado normal y si es menor a 0,9 indica obstrucción (leve entre 0,7 y 0,9; moderada entre 0,4 y 0,7 ; y severa entre 0 y 0,4$)$.

En este estudio, la medición PAS se realizó con el paciente en posición supina, luego de diez minutos de descanso y con un manguito del tamaño adecuado para cada paciente. La medición de la PAS en los miembros inferiores se realizó en forma simultánea. Mientras el médico entrenado tomaba la PAS a través del pulso de la arteria tibial posterior, el ecografista lo hacía en la pedia y viceversa. Lo mismo ocurría en los miembros superiores, pero realizando la medición en las arterias radial y humeral. Ambos operadores estaban ciegos al resultado obtenido por el otro. Cuando sólo se podía medir el pulso en una de las arterias de las piernas se tomaba esa medición para calcular el ITBPA.

\section{Resultados}

Se realizó la medición del ITBPA al $85 \%$ de la muestra, detectándose una prevalencia de EAP de $4,37 \%$. Los principales resultados se resumen en la tabla 1.

Tabla 1: características operativas* del índice tobillo brazo medido con la técnica palpatoria y tomando como test de referencia al índice tobillo brazo medido a través de ecografía doppler.

\begin{tabular}{c|c|c|c|c}
$\begin{array}{c}\text { Sensibilidad } \\
\text { (IC95\%) }\end{array}$ & $\begin{array}{c}\text { Especificidad } \\
\text { (IC95\%) }\end{array}$ & $\begin{array}{c}\text { Valor predictivo } \\
\text { negativo }\end{array}$ & $\begin{array}{c}\text { Likehood Ratio } \\
\text { positivo }\end{array}$ & $\begin{array}{c}\text { Likehood Ratio } \\
\text { negativo }\end{array}$ \\
\hline $88 \%$ & $82 \%$ & $99 \%$ & 4,98 & 0,15 \\
$(65$ a 100$)$ & $(77$ a 88$)$ & & $(3,32$ a 7,48$)$ & $(0,02$ a 0,95$)$ \\
\hline
\end{tabular}

\section{Conclusiones}

La medición del ITBPA por palpación podría ser un buen método en centros de atención primaria para detectar EAP en pacientes con riesgo CV intermedio.

Palabras claves: índice tobillo brazo, riesgo cardiovascular, ecografía doppler, aten-

Key words: ankle brachial index, cardiovascular risk, doppler ultrasonography, primary care. Fuente de financiamiento: no referida.

\section{Comentario}

La EAP es causada por arterosclerosis en las arterias de los miembros inferiores. En general presenta un periodo asintomático y aumenta la mortalidad y el riesgo de eventos CV de quienes la padecen. Puede ser fácilmente detectada mediante la realización del ITBPA, método simple, no invasivo y barato, permitiendo identificar pacientes con un alto riesgo de complicaciones CV. Por ejemplo, una revisión sistemática ${ }^{2}$ mostró que el ITBPA medido por ecografía doppler reportó que el $16,5 \%$ de los pacientes con enfermedad coronaria, el $16 \%$ de los pacientes con enfermedad cerebrovascular y el $41 \%$ de quienes mueren por causa $\mathrm{CV}$, tienen evidencia de EAP valorada por ITPBA; mientas que no la tienen cerca del $90 \%$ de los individuos que no tienen dichas condiciones. Por otro lado, siendo la EAP poco diagnosticada en ámbito de los cuidados primarios de la salud, la utilización del cálculo de ITBPA por palpación permitiría hacer diagnostico de esta condición -o por lo menos descartarlo con bastante certeza- sin la necesidad de un entrenamiento o maquinaria especial y a muy bajo costo.
Asumiendo prevalencias de EAP similares a la de la población evaluada por Migliacci y col., existe una muy baja probabilidad que un paciente con riesgo CV aumentado padezca EAP si su ITBPA por palpación es mayor a 0,9 (VPN: 99\%). Sin embargo y dado su bajo VPP (18\%) todos los pacientes con un ITBPA menor a 0,9 deberán ser evaluados con una técnica más específica como la ecografía doppler arterial de miembros inferiores con el objetivo de confirmar el diagnóstico de EAP.

\section{Conclusiones de la comentadora}

La detección de EAP asintomática mediante una tecnología sencilla y barata permitiría mejorar en el ámbito de la atención primaria la predicción del riesgo $\mathrm{CV}$, y por lo tanto, ajustar en forma más eficiente la intensidad de las intervenciones para la prevención $\mathrm{CV}$

Gabriela Buela [ Senicio de Medicina Familiar del Hospital Italiano de Buenos Aires. gabriela.buela@ hospitalitaliano.org.ar ]

Ver glosario*

Recibido el 10/06/09 y aceptado el 19/06/09

Buela G. Medición del índice tobillo brazo mediante palpación para el diagnóstico de la enfermedad arterial periférica. Evid Act Pract Ambul. $12(2)$. 58. Abr-Jun 2009. Comentado de: Migliacci y col. Ankle-brachial index measured by palpation for the diagnosis of peripheral arterial disease. Fam Pract. 2008 Aug; 25(4):228-32. PMID: Disponible bajo suscripción en URL: http://fampra.oxfordjournals.org/cgi/reprint/25/4/228

Referencia

1. Guo X y col. Sensitivity and specificity of ankle-brachial index for detecting angiographic stenosis of peripheral arteries. Heart, Lung and Blood Vessel Center, Tongji University, PR China. 2008 Apr:72(4):605-10.

2. Doobay A y col. Sensitivity and specificity of the ankle-brachial index to predict future cardiovascular outcomes: a systematic review. 2005 Jul;25(7):1463-9. Epub 2005 May 5. 